\title{
A IMPORTÂNCIA DAS BOAS PRÁTICAS DE MANIPULAÇÃO DOS ALIMENTOS AO COMBATE DE DOENÇAS: REVISÃO DE LITERATURA
}

\author{
THE IMPORTANCE OF GOOD PRACTICES IN FOOD HANDLING IN \\ THE FIGHT OF DEESEASES: LITERATURE REVIEW
}

Rhaissa Pinheiro Ferreira ${ }^{1}$

Nathália dos Reis Franco ${ }^{2}$

\begin{abstract}
RESUMO: OBJETIVOS: Descrever e analisar as produções científicas sobre a atual situaçãodos restaurantes em relação às Boas Práticas de Manipulação. MÉTODOS: O presente estudofoi executado por meio de revisão bibliográfica em línguas inglesa, portuguesa e espanhola, utilizando as bases de dados LILACS, PUBMED e SCIELO, utilizando publicações científicas dos últimos 5 anos. RESULTADOS: Foram selecionados 14 artigos que estavam dentro da temática definida e dos critérios de inclusão para realização deste estudo. De forma genérica osrestaurantes nos últimos cinco anos estão realizando as boas práticas de manipulação, precavendo-se o público de doenças originadas por alimentos contaminados, todavia até agora carecem aprimorar o aspecto higiênico. CONCLUSÃO: Consequentemente, é importante queos estabelecimentos alimentícios possam assegurar higiene sanitária apropriada e treinamento satisfatório o para os funcionários que manipulam alimentos, visando benefícios como a preservação da saúde dos consumidores.
\end{abstract}

Palavras chave: Segurança alimentar. Higiene. Serviços de alimentação. Microbiologia dos alimentos.

ABSTRACT: OBJECTIVES: Describe and analyze the scientific production about the current situation of the restaurants in relation to the Good Handling Practices. METHODS: Thepresent study was carried out through bibliographic review in English, Portuguese and Spanish, using the LILACS, PUBMED and SCIELO databases, using scientific publications from the last 5 years. RESULTS: 14 articles were selected that were within the defined theme and inclusion criteria for this study. Generically, restaurants in the last five years are performing good handling practices, preventing the public from diseases caused by contaminated food, however, until now they need

\footnotetext{
${ }^{1}$ Discente do curso de graduação em Nutrição da Universidade da Amazônia.

${ }^{2}$ Nutricionista residente da faculdade de medicina da universidade de São Paulo - Incor.
} 
to improve the hygienic aspect. CONCLUSION: Consequently, it is important that food dietary requirements ensure proper sanitary hygiene and satisfactory training for employees who handle food, benefits such as preserving consumers' health.

Keywords: Food security. Hygiene. Food services. Food microbiology. 


\section{INTRODUÇÃO}

O número de pessoas que procuram realizar sua alimentação fora de casa aumenta cada vez mais, sendo resultado das mudanças socioeconômicas que se sucederam nos últimos anos, como industrialização, urbanização, a distância entre o local de trabalho e a residência, entre outros fatores (MELLO; BACK; COLARES, 2011). Reforçando essa verificação, a Associação Brasileira das Empresas de Refeições Coletivas informou que, em 2011, foram vendidas 6 milhões de refeições ao dia e em 2014 esse número foi de 7,4 milhões, sendo o faturamento do setor de aproximadamente 13,9 bilhões de reais em 2014.

Logo, no país houve aumento significativo na quantidade de restaurantes nos últimos anos, sendo em 2013, o setor de alimentação coletiva forneceu 11,7 milhões de refeições/dia com faturamento 16,6 bilhões de reais, com predisposição ao crescimento nos anos subsequentes (ABERC, 2014). Neste contexto, se verifica um aumento na responsabilidade dos restaurantes, que devem intensificar seus controles para possibilitar a oferta de alimentos seguros, que não causem Doenças Transmitidas por Alimentos (DTA).

Segundo a RDC № 216, contaminação pode ser por "substâncias ou agentes de origem biológica, química ou física, estranhos ao alimento, que sejam considerados nocivos à saúde humana ou que comprometam a sua integridade".

O manipulador é fundamental quando se trata da segurança dos alimentos, pois, em contato com os mesmos, da origem até o momento da comercialização, pode se tornar um transmissor viável de agentes patogênicos de doenças alimentares, quando falhas e erros são cometidos. O ser humano também possui potente capacidade para veicular patógenos de pessoaa pessoa, com graves riscos à saúde (SILVA; SANTOS; SOARES, 2017).

De acordo com o Ministério da Saúde (2019), de 2009 a 2018, teve uma série histórica no total de 6.809 surtos, predominando principalmente na região sudeste, no mesmo informa que os principais agentes etiológicos responsáveis pelos surtos 
registrados são Escherichia coli, Salmonella spp, Staphylococcus aureus e Coliformes, e o principal local de ocorrência, após as residências, são os restaurantes/padarias e outras instituições(trabalho).

Segundo a Portaria 1.428, as Boas Práticas são "normas de procedimentos para atingir um determinado padrão de identidade e qualidade de um produto e/ou de um serviço naárea de alimentos, cuja eficácia e efetividade deve ser avaliada através da inspeção e/ou da investigação". Estão incluídos também produtos como bebidas, aditivos, utensílios, embalagens e todos os materiais em contato com o alimento.

A execução de boas práticas de manipulação dos alimentos em restaurantes e estabelecimentos alimentícios previne 0 acontecimentos de problemas relacionados à higiene, como o crescimento de microrganismos nos alimentos que se tornam deletérios à saúde dos comensais. Esses métodos de manipulação dos alimentos são pouco efetuados no âmbito dos restaurantes, visto que existem muitos episódios referentes à falta de higiene dos alimentos e manipuladores (CARVALHO; MORRI, 2017).

Assim, este trabalho tem como principal objetivo verificar na literatura a situação atual dos restaurantes em relação às Boas Práticas de Manipulação.

\section{METODOLOGIA}

A revisão bibliográfica de literatura é um método que tem como finalidade analisar as informações encontradas nos estudos relevantes sobre determinado tema na literatura disponível, a fim de levar a uma conclusão. Assim, é utilizado fontes científicas e fontes de divulgação de ideias, como artigos, teses, dissertações, revistas, sites, vídeos, entre outros (UNESP, 2015).

O estudo foi feito através de busca online das produções científicas nacionais e internacionais sobre a importância das boas práticas de manipulação dos alimentos em restaurantes, nas bases de dados: SCIELO - Scientific Electronic Library Online, Literatura Latino-Americana e do Caribe em Ciências da Saúde 
(LILACS) e no PubMed: MEDLINE® Retrieval on the World Wide Web Fact Sheet no período de janeiro de 2021, utilizando como questões norteadoras se as boas práticas de manipulação dos alimentos em restaurantes garantem a proteção das comidas com relação aos micro-organismos.

Para isso foram utilizados como critérios de inclusão: artigos publicados em periódicos no período de 2016 a 2021, artigos completos captados gratuitamente; textos disponíveis no idioma Português, Espanhol e Inglês. Foram excluídos do estudo dissertações, teses, monografias bem como artigos científicos repetidos. Os descritores utilizados foram respectivamente: boas práticas; higiene dos alimentos; restaurante. Foi realizada a leitura dos resumos dos artigos e foram excluídos aqueles que não atendiam ao objetivo do estudo. Após avaliação, foram excluídas 4.039 publicações, e identificados 14 artigos que preencheram todosos critérios. 
Fluxograma 1. Expandido da coleta e seleção de dados.

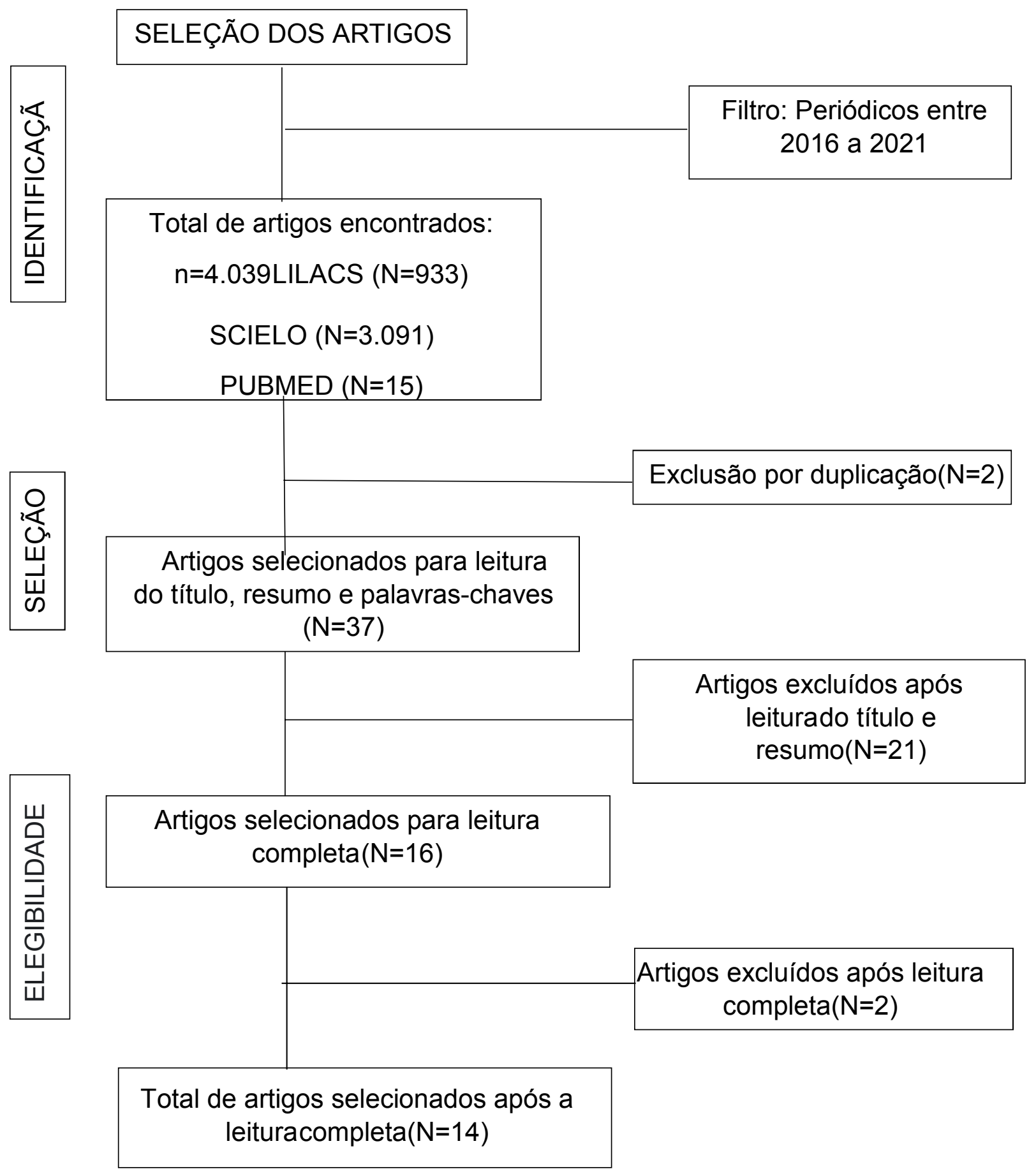

Fonte: Acervo da pesquisa, 2021. 


\section{RESULTADOS E DISCUSSÃO}

Após leitura dos resumos dos 1.513 artigos, foram selecionados 19 artigos, que foram lidos integralmente. Depois de serem analisados, 14 artigos foram selecionados como objetivo deste estudo, pois estavam em conformidade com o objetivo da pesquisa e com os critérios de inclusão. Os demais foram excluídos devido repetição, não apresentarem texto na íntegra e estarem dentro dos critérios de exclusão.

O quadro 1 foi elaborado para demonstrar quais foram os artigos estudados e seus objetivos, sintetizando de forma clara e organizada os autores, títulos, ano e objetivos de publicação dos artigos, podendo também verificar a distribuição das produções, de acordo com o ano de publicação nas referidas bases, com maior ocorrência no ano de 2018 (33,33\%) e menor número de produções, nos anos de 2018 (35,7\%), 2016 (35,7 \%), 2017 (28,5\%), 2020 (0\%) e 2021 (0\%).

Quadro 1 - Autores, bases de dados, objetivos e principais conclusões dos artigos selecionados.

\begin{tabular}{|c|c|c|c|}
\hline Autores & Título & Ano & Objetivos \\
\hline $\begin{array}{l}\text { Guimarães, } \\
\text { Ferreira, R. } \\
\text { Soares, L. S. }\end{array}$ & $\begin{aligned} & \text { B.S.; } \begin{array}{lr}\text { Perfil microbiológico de } \\
\text { densílios em unidade } \\
\text { Selimentação }\end{array} \\
& \text { nutrição comercial e } \\
& \text { institucional } \\
& \text { Salvador, BA. }\end{aligned}$ & 2018 & \begin{tabular}{llrr} 
Avaliar as & \multicolumn{1}{c}{ condições } \\
microbiológicas de utensílios \\
utilizados no processo & de \\
produção de alimentos em um \\
restaurante comercial e & um \\
institucional na cidade & de \\
Salvador, BA. & &
\end{tabular} \\
\hline $\begin{array}{l}\text { Freire, } \\
\text { al. }\end{array}$ & 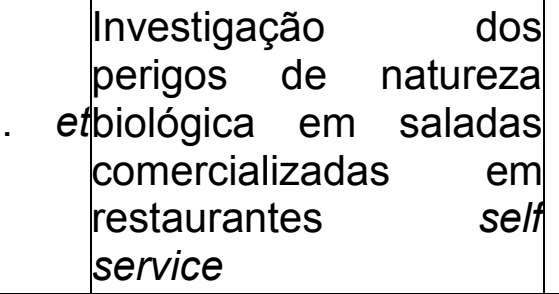 & 2018 & $\begin{array}{l}\text { Investigar os perigos denatureza } \\
\text { biológica em saladas } \\
\text { comercializadas por restaurantes } \\
\text { do tipo self service, localizados } \\
\text { no município de Mossoró, Rio } \\
\text { Grande do Norte }\end{array}$ \\
\hline
\end{tabular}




\begin{tabular}{|c|c|c|c|}
\hline Vieira, C. F.et al. & $\begin{array}{l}\text { Qualidade } \\
\text { higienicossanitária de } \\
\text { hortaliças cruas } \\
\text { servidas em restaurante } \\
\text { institucional da Baixada } \\
\text { santista, SP. }\end{array}$ & 2018 & 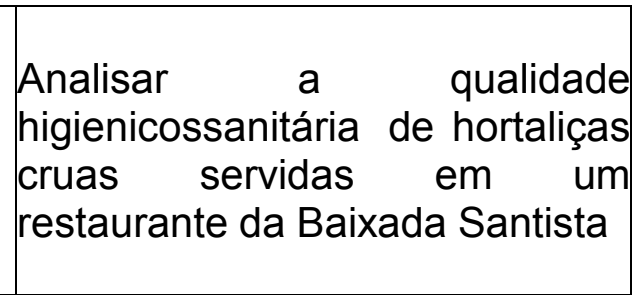 \\
\hline Pereira, L. R.et al & $\begin{array}{l}\text { Análise de perigos e } \\
\text { pontos críticos de } \\
\text { controle na produção de } \\
\text { refeições industriais. }\end{array}$ & 2018 & $\begin{array}{l}\text { controlar a segurança dos } \\
\text { alimentos, planejando o sistema } \\
\text { para evitar problemas de ordem } \\
\text { química, física ou biológica, } \\
\text { envolvendo os responsáveis em } \\
\text { tomada de decisões e registro de } \\
\text { ocorrência }\end{array}$ \\
\hline Cardoso, C. S.et & $\begin{array}{l}\text { Avaliação da qualidade } \\
\text { higienicossanitária de } \\
\text { altemakis comercializados } \\
\text { no município de Santos, } \\
\text { SP }\end{array}$ & 2018 & $\begin{array}{l}\text { análise físico-química e } \\
\text { microbiológica de temakis } \\
\text { servidos em restaurantes } \\
\text { especializados do município de } \\
\text { Santos/SP }\end{array}$ \\
\hline $\begin{array}{l}\text { Silva, } \quad \text { R.N.A. } \\
\text { Santos, } \\
\text { Soares, } \\
\text { S.S.S.S. }\end{array}$ & $\begin{array}{l}\text { Avaliação } \\
\text { microbiológica das } \\
\text { mãos } \\
\text { manipuladoresem } \\
\text { restaurantes } \\
\text { comerciaise } \\
\text { institucionais da cidade } \\
\text { deSalvador, BA. }\end{array}$ & 2017 & \begin{tabular}{lrr} 
Realizar & a & \multicolumn{2}{c}{ avaliação } \\
microbiológica das & mãos de \\
manipuladores de & restaurantes \\
comerciais e institucionais da \\
cidade de Salvador/BA, pormeio \\
da pesquisa de & Staphylococcus \\
aureus & e & coliformes \\
termotolerantes & (Escherichia \\
coli). & \\
\end{tabular} \\
\hline $\begin{array}{l}\text { Moreira, R. C. } \\
\text { Dutra, A. }\end{array}$ & $\begin{array}{l}\text { A oferta de alimentos } \\
\text { seguros em restaurante } \\
\text { comercial do tipo self } \\
\text { service. }\end{array}$ & 2017 & $\begin{array}{l}\text { Avaliar a oferta de alimentos } \\
\text { seguros em um restaurante } \\
\text { comercial do tipo self service da } \\
\text { cidade do Rio de Janeiro }\end{array}$ \\
\hline $\begin{array}{l}\text { Panetta, M. H. } \\
\text { al. }\end{array}$ & $\begin{array}{l}\text { Restaurante } \quad \text { com } \\
\text { autosserviço: } \\
\text { etcomportamento r do } \\
\text { consumidor x risco de } \\
\text { contaminação } \\
\text { alimentos. }\end{array}$ & 2017 & $\begin{array}{l}\text { Analisar o comportamento do } \\
\text { consumidor e o risco de } \\
\text { contaminação dos alimentos em } \\
\text { balcão de distribuição de } \\
\text { restaurantes com autosserviço } \\
\text { (self service). }\end{array}$ \\
\hline $\begin{array}{l}\text { Simon, } \\
\text { Benedetti, V. P. }\end{array}$ & $\begin{array}{l}\text { Avaliação } \\
\text { contaminação } \\
\text { microbiológica } \\
\text { esponjas utilizadas em } \\
\text { serviços de alimentação } \\
\text { da cidade de } \\
\text { Marmeleiro - PR. }\end{array}$ & 2016 & $\begin{array}{l}\text { Fazer uma avaliação da } \\
\text { contaminação microbiológica e } \\
\text { dois procedimentos de } \\
\text { desinfecção de esponjas } \\
\text { utilizadas em serviços de } \\
\text { alimentação. }\end{array}$ \\
\hline
\end{tabular}




\begin{tabular}{|c|c|c|}
\hline $\begin{array}{ll}\text { Condições } & \\
\text { higienicossanitárias } & \text { de } \\
\text { preparações r com } \\
\text { vegetais crus em } \\
\text { restaurantes tipo self } \\
\text { service no município de } \\
\text { Alfenas - MG. }\end{array}$ & 2016 & $\begin{array}{l}\text { Avaliar as r condições } \\
\text { higienicossanitárias } r \text { de } \\
\text { preparações com vegetais crus } \\
\text { em restaurantes tipo self service } \\
\text { no município de Alfenas - MG, a } \\
\text { fim de se detectar possíveis } \\
\text { micro-organismos patogênicos. }\end{array}$ \\
\hline 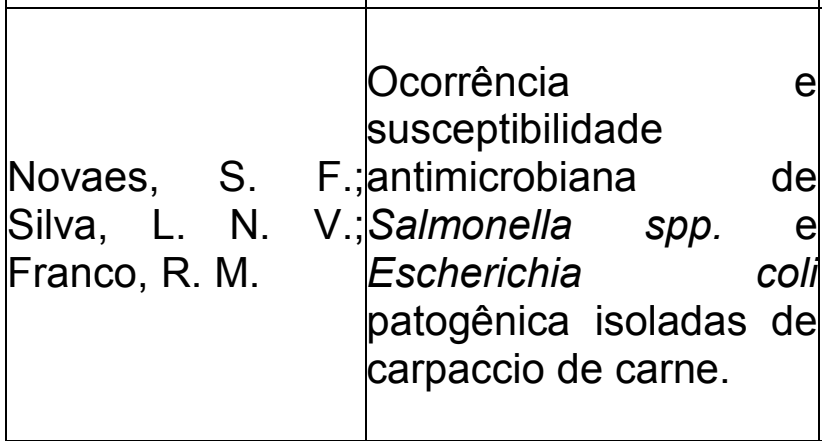 & 2016 & $\begin{array}{l}\text { Ocorrência de estirpes de } \\
\text { Salmonella spp. e E.coli } \\
\text { patogênicas em carpaccios de } \\
\text { carne comercializados em bares } \\
\text { e restaurantes e avaliar o perfil } \\
\text { de resistência aos agentes } \\
\text { antimicrobianos utilizados na } \\
\text { rotina do tratamento de } \\
\text { infecções alimentares }\end{array}$ \\
\hline 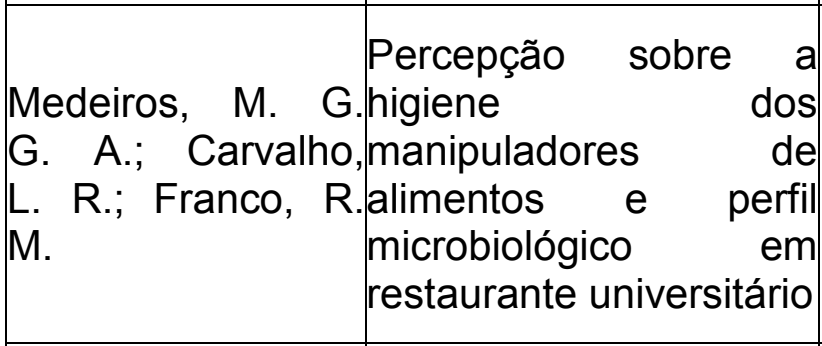 & 2017 & $\begin{array}{l}\text { Analisar a percepção dos } \\
\text { manipuladores de alimentos em } \\
\text { relação às práticas de higiene no } \\
\text { local de trabalho e a } \\
\text { correlacionar com os resultados } \\
\text { das análises bacteriológicas e } \\
\text { das observações sistematizadas }\end{array}$ \\
\hline \begin{tabular}{|l|l|} 
& \multicolumn{2}{l}{ Qualidade } \\
higienicossanitária de \\
Dreckmann, M. V. \\
sushis e sashimis \\
et al. & $\begin{array}{l}\text { comercializados em } \\
\text { restaurantes orientais } \\
\text { de balneário Camboriú, } \\
\text { SC }\end{array}$ \\
\end{tabular} & & $\begin{array}{l}\text { Avaliar a } \quad \text { condição } \\
\text { higienicossanitária dos produtos } \\
\text { sushi e sashimi comercializados } \\
\text { em restaurantes orientais, } \\
\text { considerando os serviços self } \\
\text { service e à la carte }\end{array}$ \\
\hline \begin{tabular}{|l|lr} 
Teixeira, A. C. Et & $\begin{array}{l}\text { Boas práticas na } \\
\text { manipulação }\end{array}$ \\
alimentos de \\
restaurantes comerciais \\
self service
\end{tabular} & & $\begin{array}{l}\text { Avaliar as condições } \\
\text { higienicossanitárias } r \text { em } \\
\text { restaurantes comerciais do tipo } \\
\text { self service localizados no } \\
\text { município de Vila Velha - ES. }\end{array}$ \\
\hline
\end{tabular}

Fonte: Acervo da pesquisa, 2021.

A partir das análises dos artigos percebeu-se que todos os artigos são da Revista Cientifica em Ciências da Saúde, e que a contaminação dos alimentos pode ocorrer depois da sua preparação, isto é, no momento da distribuição até mesmo pelos próprios comensais. Foi possível notar também que alguns métodos de higiene, feito pelos colaboradores, quando bem treinados e efetuados são bastante eficazes quanto à preservação dos alimentos. De modo geral, os restaurantes nos últimos cincos anos estão precisando de mais instruções de boas práticas. Vale 
ressaltar que esses métodos de higiene quando são supervisionados por técnicos como tecnólogos de alimentos, nutricionistas entre outros profissionais são executados corretamente.

Segundo Panetta, M. H. et al. (2017) em um estudo observacional em dois restaurantes dentro de um Shopping em São Paulo, onde foi observado que o comportamento, dos comensais, de maior incidência, foi o de falar, rir ou espirrar enquanto se servia, analisam os dois estabelecimentos em conjunto, este comportamento apresentou $62,5 \%$ de ocorrência. Porem essa atitude podem propiciar a contaminação por Staphylococcus aureus. Também como, outra ação encontrada com frequancia foi de mexer nos cabelos, nariz, orelha e depois se servir. Com tudo, essas ações transportam vários micro-organismos encontrados nas mãos, nariz, boca e cabelo, como Staphylococcus aureus, Encherichia coli, Bacillus cereus e Pseudomonas aeruginosa podem ser transferidos para os alimentos.

A partir das analises dos artigos percebeu-se que todos os artigos é da Revista Cientifica em Em um estudo transversal realizado por Guimarães, B.S.; Ferreira, R. S.; Soares, L. S. (2018) mostrou que os utensílios das duas cozinhas avaliados, são eles bandeja, cortador/faca, pegador, placa de corte verde (hortaliças) e gastronorm, na pesquisa se encontravam em condições higiênicas insatisfatórias, no qual verifica-se que todos os utensílios estavam contaminados por, no mínimo, um tipo de micro-organismo, os mesmos eram mesófilos aeróbios, Estafilococos coagulase positivo, Coliformes totais e E.coli. Segundo Simon, D.; Benedetti V. P. (2016) um veículo disseminador de micro-organismos pode ser as esponjas, utilizadas para lavar a louça. Além do mais, na sua pesquisa em 7 restaurantes foi encontrado $77,77 \%$ das esponjas analisadas estavam contaminadas por coliformes totais e $44,44 \%$ por coliformes termotolerantes, indicando maior probabilidade de risco à saúde por contaminação cruzada e a não utilização de procedimentos higienicossanitários corretos. Contudo, a fervura por cinco minutos reduziu 99,99\% das bactérias nas esponjas.

Posteriormente um estudo transversal desenvolvido com amostra de 24 mãos dos colaboradores de 5 restaurantes comerciais e 6 restaurantes institucionais, localizado na cidade de Salvador/BA, no qual avaliaram a presença de 
Staphylococcus aureus e coliformes termotolerantes (Escherichia coli). Nesse sentido, os restaurantes institucionais 2, 3, 4 e 6 apresentaram todas as amostras acima do valor padrão de referência de $10^{2}$ UFC/mão, para os dois microorganismos, em quando os restaurantes comerciais 1, 2, 3, e 4 apresentaram todas as amostras com valores dentro dos limites aceitáveis. Vale ressaltar que nos restaurantes 1 e 4 os manipuladores encontravam- -se no limite da faixa aceitável que é $10^{2}$ UFC/mão e apenas o 5 apresentou-se fora do padrão de referência (SILVA, R.N.A.; SANTOS, A.P.L.; SOARES, L.S.S.,2017).

A deficiência na higienização das mãos, EPI (equipamento de proteção individual) e utensílios, é reveladora de uma grave distorção entre um possível conhecimento sobre corretaspráticas de higiene no local de trabalho, em relação aos hábitos e atitudes inadequadas do manipulador de alimentos (MEDEIROS, M. G. G. A.; CARVALHO, L. R.; FRANCO, R. M.,2017).

Logo, em uma pesquisa realizada com dez estabelecimentos comerciais do tipo selfservice deve como objetivo a investigação dos perigos de natureza biológica em saladas cruas, onde ocorreu a análises parasitárias, pesquisa de coliformes e determinação de Escherichia colie Salmonella sp. Logo, de acordo com a análise realizada foi observado que as saladas comercializadas se apresentaram em conformidade mostrando índices de coliformes a $45^{\circ} \mathrm{C}$ dentro do aceitável, ausência de $E$. coli e Salmonella $s p$, no entanto ocorreram falhas no processamento do alimento (FREIRE, B.C.F. ET AL,2018).

Outrossim, realizado por Silva, A. A. et al. (2016) com 6 restaurantes tipo self service também avalizando as saladas cruas a fim de se detectar possíveis microorganismos patogênicos, as amostras foram submetidas à determinação de coliformes a $35^{\circ} \mathrm{C}$ e $45^{\circ} \mathrm{C}$, contagem de bactérias aeróbias mesófilas, Staphylococcus coagulase positiva e Salmonella sp. Neste estudo verificou-se a presença de coliformes a $35^{\circ} \mathrm{C}$ nas 18 (100\%) amostras analisadas, não houve resultado positivo para Staphylococcus e 7 (38,8\%) apresentaram resultados positivos para Salmonella $s p$. Com tudo, os resultados indicam a ocorrência de falha nos processos de sanitização, higiene inadequada durante a manipulação.

Em um outro estudo por Vieira, C. F.et al. (2018) que também foi feito com saladas cruas de restaurantes, os mesmos fizeram a análise microbiológica 
(Coliformes a $45^{\circ} \mathrm{C}$ e Salmonella sp/25g.) e aplicação do checklist nas instalações do restaurante, no qual as coletas foram feitas por 4 meses no período do almoço e janta, totalizando 30 amostras. Logo, constatou-se que a qualidade que a qualidade da higienicossanitária das hortaliças cruasservidas no restaurante não foi satisfatória e a boas práticas observadas comprometeram a qualidade das refeições servidas, o que poderia comprometer a saúde dos consumidores, sendoassim se tornando um meio de propagação de doenças transmitidas por alimentos.

O estudo de Moreira, R. C.; Dutra, A. (2017) em um restaurante self service, foram coletadas 185 amostras, sendo 34 de preparações quentes (acompanhamentos e guarnições), 23de carnes, 19 de saladas cruas, 23 de saladas cozidas, 11 de refrescos, 20 de água, 11 swabs de mãos de manipuladores de alimentos e 44 swabs de utensílios, onde foram encontrados. Encontrou-se inadequação em relação à contagem de Coliformes a $45^{\circ} \mathrm{C}$ em amostras de preparações quentes, carnes, saladas cozidas e cruas, sugerindo condições higiênicas inadequadas durante a preparação e/ou recontaminação após o preparo, o que pode ter ocorrido por contaminação cruzada, através de equipamentos sujos, manipulação sem cuidados de higiene ou contato com outros alimentos em prépreparo.

Para Pereira, L.R. et al (2018) as informações encontradas a partir do programa Análise de Perigos e Pontos Críticos de Controle -APPCC em um restaurante industrial para melhorar a segurança do alimento por meio da determinação dos Pontos Críticos de Controle - PCC desde o recebimento da matéria-prima até sua distribuição e análise microbiológica, com coliformes totais e a $45^{\circ} \mathrm{C}$ e Staphylococcus coagulase positiva. A determinação dos PCC contribui com a qualidade das refeições produzidas no restaurante estudado pois pode prevenir, reduzir ou eliminar um perigo a nível aceitável.

Cardoso, C. S et al (2018) fez um estudo com temakis de salmão de dez estabelecimentos diferentes, as análises feitas neles foi de Coliformes a $45^{\circ} \mathrm{C}$; Estafilococos coagulase positiva; Vibrio parahaemolyticus e Salmonella sp. Verificouse, em $20 \%$, a presença de coliformes e em um com a presença de estafilococos em quantidades de micro-organismos/g acima do limite, uma amostra estava com aceitável determinado pela Agência Nacional de Vigilância Sanitária - ANVISA. Os 
testes bioquímicos de Vibrio parahaemolyticus foram negativos para confirmação da espécie. No entanto, foi possível identificar as seguintes bactérias:Chromobacterium violaceum, Burkholderia cepacia, Pseudomonas stutzeri ePseudomonas fluorescens e não foi encontrado Salmonella sp nessa análise, seis amostras apresentaram colônias suspeitas, as quais foram submetidas aos testes bioquímicos que indicaram a presença de Escherichia coli, Klebsiella ozaenae e Klebsiella rhinoscleromatis.

Em outro estudo também realizado com comida oriental analisou sushi e sashimi de 5 restaurantes distintos, na qual a análise análises microbiológicas de Coliformes a $45^{\circ} \mathrm{C} / \mathrm{g}$ (coliformes fecais), Estafilococos coagulase positiva (Staphylococcus aureus), Vibrio parahaemolyticus e Salmonella sp. Dentre os resultados obtidos, para as bactérias Salmonella $\mathrm{sp}$ e Vibrio parahaemolyticus, verificou-se ausência de crescimento de colônias características em todas as amostras, em quando análise de Staphylococcus aureus apresentou crescimento de colônias atípicas e presença de colônias típicas e o mesmo ocorreu para coliformes a $45^{\circ} \mathrm{C} / \mathrm{g}$ (DRECKMANN, M. V. ET AL.,2016).

Portanto, com a alta existência de é necessária uma maior preocupação dos consumidores, dos manipuladores de alimentos e dos órgãos fiscalizadores com fatores relacionados à qualidade da matéria-prima, às condições de preparo e higiene dos estabelecimentos (NOVAES, S. F.; SILVA, L. N. V.; FRANCO, R. M.,2016). Mas também, as principais inadequações observadas estavam relacionadas às condições das edificações, instalações, manipulação, armazenamento e exposição dos alimentos (TEIXEIRA, A. C. ET AL.,2016).

\section{CONSIDERAÇÕES FINAIS}

Com base nas leituras apresentadas, ficou evidente que os restaurantes precisam aplicar mais as boas práticas de manipulação, usando métodos eficazes de higiene e fazendo capacitação com os funcionários, para poder garantir qualidade aos consumidores e evitando que ocorram surtos decorrentes de alimentos contaminados por microrganismos, já outros mostraram poucas garantias de higiene, necessitando de maior rigor nas fiscalizações. É importante preconizar que 
é sempre essencial a utilização e renovação constante de meios que garantam a qualidade dos alimentos servidos, e vale ressaltar que esses métodos de higiene quando são supervisionados por técnicos como nutricionistas.

Conclui-se que um treinamento apropriado para os funcionários que manipulam alimentos é extremamente necessário, pois são os responsáveis pela higienização correta dos insumos que serão consumidas e por evitar a contaminação cruzada com outros alimentos. É importante reforçar este treinamento periodicamente e orientar os funcionários da unidade de alimentação e nutrição sobre a relevância deste, para garantir assim que as boas práticas sejamadotadas e consequentemente, reduzir o risco para a saúde do consumido.

\section{REFERÊNCIAS BIBLIOGRÁFICAS}

ABERC. Associação Brasileira das Empresas de Refeições Coletivas. Mercado real. Estimativa 2014.

BRASIL. Ministério da Saúde. Secretaria de Vigilância Sanitária. Portaria n. 1.428, de 26 de novembro de 1993. Regulamento técnico sobre condições higiênicos-sanitárias e de boas práticas de fabricação para estabelecimentos produtores/ industrializadores de alimentos. Diário Oficial [da] República Federativa do Brasil, Brasília. Disponível em: < https://www.in.gov.br/materia/-/asset_publisher/Kujrw0TZC2Mb/content/id/57496468/do1- 201812-31-portaria-n-1-428-de-28-de-dezembro-de-2018-57496251>. Acesso em:24 jan.2021.

BRASIL. Ministério da Saúde. Surtos de Doenças Transmitidas por Alimentos no Brasil,

https://portalarquivos2.saude.gov.br/images/pdf/2019/fevereiro/15/Apresenta o-Surtos-DTAFevereiro-2019.pdf. Acesso em 07 jan 2021.

CARVALHO, S. J. E. J.; MORI, E. A importância das boas práticas de manipulação dos alimentos em restaurantes: revisão integrativa da literatura. Rev. e-ciência. V. 5, n. 2, p. 108115, 2017. Disponível em: < http://www.revistafjn.com.br/revista/index.php/eciencia/article/view/269/pdf_269>. Acesso em:10 jan 2021

MELLO, A. G.; BACK, F. S.; COLARES, L. G. T. Condições higiênico-sanitárias de restaurantes self-services localizados no estado do Rio de Janeiro. Hig. Alim. Rio de Janeiro, v. 25, n. 2, p. 64-9, 2011.

SILVA, R. N. A.; SANTOS A. P. L.; SOARES, L. S. Avaliação microbiológica das mãos de manipuladores em restaurantes comerciais e institucionais da cidade de Salvador, BA. Higiene Alimentar - Vol.31 - $n^{\circ}$ 270/271 - Julho/Agosto de 2017. Disponível em: < https://pesquisa.bvsalud.org/portal/resource/pt/biblio-848950>. Acesso em:10 jan 2021.

UNESP. (Botucatu). Tipos de revisão de literatura. 2015. Disponível em: <https://www.fca.unesp.br/Home/Biblioteca/tipos-de-evisao-de-literatura.pdf>. Acesso em: 28 out. 2020. 